\title{
Molecular Signatures of the Aging Brain: Finding the Links Between Genes and Phenotypes
}

\author{
Giuseppe Lupo ${ }^{1}$ (D) Silvana Gaetani ${ }^{2} \cdot$ Emanuele Cacci $^{3} \cdot$ Stefano Biagioni $^{3} \cdot$ Rodolfo Negri $^{3}$
}

Published online: 3 June 2019

(C) The American Society for Experimental NeuroTherapeutics, Inc. 2019

\begin{abstract}
Aging is associated with cognitive decline and increased vulnerability to neurodegenerative diseases. The progressive extension of the average human lifespan is bound to lead to a corresponding increase in the fraction of cognitively impaired elderly individuals among the human population, with an enormous societal and economic burden. At the cellular and tissue levels, cognitive decline is linked to a reduction in specific neuronal subpopulations, a widespread decrease in synaptic plasticity and an increase in neuroinflammation due to an enhanced activation of astrocytes and microglia, but the molecular mechanisms underlying these functional changes during normal aging and in neuropathological conditions remain poorly understood. In this review, we summarize very recent and outstanding progress in elucidating the molecular changes associated with cognitive decline through the genome-wide profiling of aging brain cells at different molecular levels (genomic, epigenomic, transcriptomic, proteomic). We discuss how the correlation of different molecular and phenotypic traits driven by mathematical and computational analyses of large datasets has led to the prediction of key molecular nodes of neurodegenerative pathways, and provide a few examples of candidate regulators of cognitive decline identified with these approaches. Furthermore, we highlight the dysregulation of the synaptic transcriptome in neuronal cells and of the inflammatory transcriptome in glial cells as some of the key events during normal and neuropathological human brain aging.
\end{abstract}

Key Words Brain $\cdot$ Aging $\cdot$ Cognitive decline $\cdot$ Gene expression $\cdot$ DNA methylation $\cdot$ Histone acetylation

\section{Introduction}

In the mammalian brain, aging is associated with progressive cognitive decline and with enhanced susceptibility to late onset neurodegenerative diseases. The cognitive deficits linked to normal and pathological aging are becoming widespread in the human population, mainly due to its increasing average life expectancy. Consequently, the economic and societal

Electronic supplementary material The online version of this article (https://doi.org/10.1007/s13311-019-00743-2) contains supplementary material, which is available to authorized users.

Giuseppe Lupo

giuseppe.lupo@uniroma1.it

1 Department of Chemistry, Sapienza University of Rome, Piazzale A. Moro, 00185 Rome, Italy

2 Department of Physiology and Farmacology "V. Erspamer", Sapienza University of Rome, Piazzale A. Moro, 00185 Rome, Italy

3 Department of Biology and Biotechnology “C. Darwin”, Sapienza University of Rome, Piazzale A. Moro, 00185 Rome, Italy burden of age-related neurological conditions is increasingly growing and difficult to sustain. This has prompted extensive efforts to investigate the mechanisms underlying the functional impairment of the aged brain, to develop novel therapeutic approaches able to slow down the cognitive decline of the elderly population and to prevent/treat age-related neuropathologies.

At the cellular level, aging is not linked to an overall reduction in the amount of pre-existing neurons in the central nervous system, although certain brain structures and neuronal subtypes may be more vulnerable than others and selectively prone to cell loss $[1,2]$. For example, age-related neuronal death has been described in specific subpopulations of the cerebral cortex and the cerebellum $[3,4]$. The strongest effects of aging, however, appear to be exerted at the level of synaptic activity, as shown by the age-associated decline in the number of synapses and in synaptic plasticity in several brain regions [5-8]. Moreover, a landmark of the aged brain is an increased level of neuroinflammation, which can contribute to the alterations in neuronal/synaptic function [9]. All these deficits worsen in age-related neurodegenerative conditions, such as 
late onset Alzheimer's disease (AD), although there are also peculiar differences between normal and neuropathological aging $[2,9,10]$. Finally, aging has a major impact on the generation of new neurons in the adult brain. At least in rodents, in which adult neurogenesis has been best characterized, a sharp age-dependent drop in the production of new neurons is detectable at the level of both of the main adult neurogenic niches, the subventricular zone (SVZ), flanking the lateral ventricles, and the hippocampal subgranular zone (SGZ) $[11,12]$. Thus, aging can hinder the remodelling of neural circuits by affecting synaptic connectivity and the integration of new neuronal elements, leading to a progressive decline in the functional plasticity of the brain and in cognitive abilities.

Very recently, several studies have investigated the molecular mechanisms underlying the functional changes taking place in the aging brain, by exploiting genome-wide nextgeneration sequencing (NGS) technologies to dissect the age-related molecular signatures of different brain cell types. In this review, we summarize the main experimental approaches that have been employed so far and the common themes emerging from the complexity of these highthroughput analyses. We focus on cell type-specific, regionspecific, and age-specific misregulation of genes related to neuronal/synaptic activity, immune response/inflammation, autophagy/lysosome function, cell death/stress regulation, and cell signalling as some of the molecular events implicated in normal and pathological brain cell aging. Furthermore, we discuss how the integrated analyses of several NGS datasets is starting to shed light on the genetic/epigenetic mechanisms underpinning these molecular alterations and the causative links between age-related changes in gene activity and cellular phenotypes.

\section{Molecular Profiling of Aged Brain Cells: Insights from Genome-Wide Transcriptomic and Epigenomic Studies}

Over the last 15 years, several transcriptomic datasets from human and rodent samples of different ages and brain regions have been reported. In the cases of human studies, the cohorts of individuals used for these analyses have often included subjects with clinical diagnosis of AD at the time of sampling, thus providing insights into the mRNA changes associated with both normal and neuropathological aging. Several genome-wide analyses of age-related epigenetic modifications, especially DNA methylation and histone acetylation, have also been described, although the available epigenomic datasets are still limited, when compared with transcriptomic resources. Recently, the shift from microarray-based to NGSbased approaches and the processing of larger numbers of samples have enhanced the power and resolution of these studies. This has shifted the focus from detecting transcriptional/epigenetic alterations in a few genes or gene categories to deciphering coordinated changes in broad genomic regions influencing multiple biological processes, at the same time bringing alternative splicing and moderately expressed non-coding RNAs into the picture. Another critical breakthrough made possible by the improved technological platforms is the gradual transition from bulk tissue to cell type-specific datasets, unveiling more subtle, but functionally relevant, changes in gene activities. Crucially, these investigations now routinely include a detailed phenotypic characterization of the tissue samples used for the molecular analyses, and a long-term cognitive profile of the tissue donors, thus allowing to correlate transcriptomic and epigenomic changes with cellular and cognitive alterations. The frontier of this approach is represented by single-cell NGS analyses. On the one hand, single-cell transcriptomics can reveal gene signatures associated with aging in distinct neural stem/progenitor cell (NSPC) subpopulations [13]. On the other hand, singleneuron whole genome sequencing has been recently used to analyze the accumulation of somatic mutations with aging in humans, a phenomenon named genosenium, which shows age-related, region-related, and disease-related molecular signatures [14].

Altogether, this work has already provided extensive evidence that both normal and neuropathological aging, as well as the associated functional modifications of brain cells, correlate with broad changes at the transcriptomic and epigenomic levels. In spite of the large variability among different studies due to significant differences in the sourced samples and in the experimental approaches used, consistent patterns of age-related gene regulation are starting to emerge, raising the hopes of eventually explaining the age-dependent cognitive decline at the molecular level. Yet, a clear association between a transcriptional/epigenetic trait and a phenotypic trait does not provide a causal relationship between them, because the molecular change could lie upstream or downstream of the associated phenotype. Another critical and partially overlapping issue is how many of the observed gene expression and epigenetic changes associated with age are actually related to the intrinsic genotypic variation and how many can be rather ascribed to extrinsic non-genetic influences. Clearly, functional studies in appropriate experimental settings (e.g., gain- and loss-of-function approaches in vitro and in vivo) are mandatory to prove the causative roles of key genes and molecular pathways in cognitive decline. Given the huge number of molecular changes correlating with age, however, a systematic functional characterization of genes/ pathways needs to be preceded by strategies to select the candidates most likely to act upstream of cognitive impairment. In the next section, we will highlight recent high-throughput studies in human subjects showing how it is possible to integrate several large-scale datasets (genomic, transcriptomic, 
epigenomic, proteomic, phenotypic) to make predictions about the molecular nodes driving cognitive decline. In the following sections, we will focus on the key age-related molecular changes revealed in specific cell types and regions of the human brain. Recent studies using mouse models to analyze the genome-wide transcriptomic and epigenomic modifications occurring in NSPCs of the aging neurogenic niches have been reviewed elsewhere [12].

\section{Harnessing High-Throughput Human Brain Datasets to Identify Candidate Regulatory Pathways of Cognitive Decline}

In 4 related studies, Mostafavi, Tasaki, Klein, De Jager, Bennett, and co-authors [15-18] have recently used different bioinformatic pipelines to analyze genome-wide transcriptomic [15], DNA methylation [19], and histone H3 lysine 9 acetylation (H3K9ac) [18] datasets from dorsolateral prefrontal cortex (DLPFC) postmortem samples of longitudinal cohorts of human subjects of different ages. Donors had different degrees of cognitive decline and neuropathological traits, such as amyloid beta (Aß) levels, hyperphosphorylated Tau (pTau) levels, and clinical diagnosis of AD, which had been characterized at the time of tissue sampling. These molecular and phenotypic datasets were also superimposed on known genomic maps of single nucleotide polymorphisms (SNPs) associated with cognitive decline and/or AD in available genome-wide association studies (GWAS).

In one study [15], the above described datasets were analyzed using the module-trait network (MTN) method, mining them for modules of co-expressed genes that were then ranked based on their association with cognitive decline and neuropathological $\mathrm{AD}$-related traits (e.g., $\mathrm{A} ß$ and/or pTau burden). This analysis pointed to a module of 390 co-expressed genes (named module 109) as the one most strongly associated with cognitive impairment. Genes within this module were then prioritized to identify the most likely candidates upstream of cognitive-related phenotypes on the basis of several parameters, including gene network connectivity, gene-phenotype association, and expression levels in astrocyte and neuron in vitro cultures. Twelve of the top hits in this module (Table 1) were selected to analyze the levels of their protein products in DLPFC samples by means of selection reaction motif (SRM) quantitative proteomics, revealing associations between protein levels and cognitive decline for several of them [20]. Two of the top hits, the semaphorin receptorencoding gene $P L X N B 1$ and INPPL1, a gene involved in the modulation of insulin/insulin growth factor (IGF) signalling pathways, were further validated by functional assays. Both genes were found to be expressed at the protein level in astrocytes of the frontal cortex in AD patients, and their knockdown in primary astrocyte in vitro cultures reduced the extracellular levels of Aß42, confirming a role upstream of neuropathological phenotypic traits [15]. Further functional work will be needed to assess the false positive rate of the MTN approach and to reveal roles of the candidate genes in aspects of cognitive decline other than $\mathrm{A} \beta 42$ accumulation.

Another study [16] employed the above described multiomic (mRNA, DNA methylation, H3K9ac, SNPs) datasets to perform a global correlation analysis among transcription, epigenetic marks, and genomic variants. This analysis was aimed at mining genes, whose expression levels were significantly associated with SNPs and with changes in either DNA methylation or H3K9ac patterns. The molecular data were then superimposed on phenotypic datasets to define, for each of the genes meeting the above criteria, a local regulatory network (LRN), predicting causal relationships (i.e., an upstream, downstream, or independent hierarchical level in the network) among mRNA levels, epigenetic modifications, and phenotypes. This pipeline allowed the identification of a subset of genes predicted to act upstream of cognitive decline, which were further prioritized based on additional parameters, including the association with cognitive deficits/AD according to independent GWAS analyses. Notably, the top hits resulting from this work (Table 1) included $S E M A 3 F$, encoding for a semaphorin family member that has been implicated in the regulation of PI3K and AKT kinases, key intracellular transducers of the insulin/IGF signalling pathway [16, 21]. No functional experiments, however, were performed to validate the identified candidate genes. In future work, it would be important to implement this approach by including additional omic datasets (e.g., genome-wide profiling of miRNAs and additional epigenetic modifications) and by extending single gene LRNs to multi-gene networks featuring the interactions between different genes. Furthermore, for both MTN and LRN approaches, follow-up studies will benefit from the analysis of other brain regions and of additional cohorts of subjects with different ancestry composition and education levels.

A third study [17] took advantage of available GWAS datasets providing $457 \mathrm{SNPs}$ correlated to $\mathrm{AD}$, to define a polygenic risk score associated with $\mathrm{AD}$ (AD-PRS) for each individual in longitudinal cohorts of aging human subjects genotyped for the AD-related SNPs. To evaluate AD-PRS correlations with specific phenotypic and molecular traits, this analysis was then integrated with phenotypic profiles, the abovementioned multi-omic datasets and SRM proteomic quantification in DLPFC samples for a group of proteins potentially linked to AD. As expected, a high AD-PRS correlated with cognitive decline and neuropathological phenotypes (e.g., $\mathrm{A} \beta$ and pTau burden). Remarkably, this pipeline also revealed the association of AD-PRS with distinct molecular features, including the expression levels of the IGFBP5 protein, a key regulator of insulin/IGF signalling encoded by one of the candidate genes in the above described, cognitive-related, gene module (module 109) [15, 17]. 
Table 1 Candidate regulators of cognitive decline identified through genome-wide molecular profiling of human brain cells. Representative candidate genes associated with cognitive decline were identified through moduletrait network, local regulatory network, or key driver analyses. For the genes presented in bold, the association with neuropathological traits (e.g., increased $A \beta$ and/or pTau levels) was further validated by means of functional assays in cell cultures (see text for further details and references)

\begin{tabular}{lcc}
\hline Module-trait network analysis & Local regulatory network analysis & Key driver analysis \\
\hline AK4 (adenylate kinase 4) & C1QTNF4 (Clq and TNF & CCT5 (chaperonin \\
& related 4) & containing TCP1 subunit 5) \\
ANKRD40 & DHRS11 & COMT (catechol-O- \\
(ankyrin repeat domain 40) & (dehydrogenase/reductase 11) & methyltransferase) \\
BCL2L1 (BCL2 like 1) & NPM3 & GNA12 (G protein subunit \\
& (nucleophosmin/ & alpha 12) \\
FBXO2 (F-box protein 2) & nucleoplasmin 3) & HSPA2 (heat shock protein \\
& transcriptional regulator) & family A member 2) \\
HSPB2 (heat shock protein & RABEP2 (rabaptin, RAB GTPase & PDHB (pyruvate dehydrogenase \\
family B member 2) & binding effector protein 2) & E1 beta subunit) \\
IGFBP5 (insulin line growth & SCG3 (secretogranin III) & RGS4 (regulator of G protein \\
factor binding protein 5) & & signaling 4) \\
INPPL1 (inositol polyphosphate & SEMA3F (semaphorin 3F) & ST18 (C2H2C-type zinc finger \\
phosphatase like 1) & & transcription factor) \\
ITPK1 (inositol- & SLC22A23 (solute carrier & \\
tetrakisphosphate 1-kinase) & family 22 member 23) & \\
KIF5B (kinesin family & STAU1 (staufen double-stranded & \\
member 5B) & RNA binding protein 1) & \\
PLXNB1 (plexin B1) & TRIOBP (TRIO and F-actin & \\
SASH1 (SAM and SH3 & binding protein) & \\
domain containing 1) & & \\
SLC6A12 (solute carrier & & \\
family 6 member 12) & & \\
VAT1 (vesicle amine transport 1) & & \\
& &
\end{tabular}

Building up on the same multi-omic datasets and especially focusing on $\mathrm{H} 3 \mathrm{~K} 9 \mathrm{ac}$ profiling, a fourth study [18] mapped the age-associated genome-wide changes in H3K9ac deposition in the human DLPFC and compared them with transcriptional and phenotypic patterns, identifying genomic segments in which $\mathrm{H} 3 \mathrm{~K} 9 \mathrm{ac}$ distribution was linked with transcriptional activity and with Tau-related phenotypic traits. The authors then screened drug repurposing databases to mine for compounds associated with gene expression signatures that were predicted to negatively correlate with the transcriptional profiles of Tau-related H3K9ac domains. This led to the identification and functional validation of a small molecule inhibitor of the heat shock protein HSP90, which could attenuate some of the effects of Tau overexpression in neuronal in vitro cultures [18]. Notably, the gene encoding for another heat shock protein, HSPB2, was part of the cognitive-related gene module (module 109) identified by MTN analysis [15]. Moreover, increased levels of HSPB2 were shown to be associated with cognitive impairment by proteomic assays in DLPFC samples [20]. Remarkably, a different study also disclosed a heat shock protein, HSPA2, as a candidate key driver of AD-related neuropathology [22]. This conclusion was reached by means of a pipeline that included the identification of modules of differentially co-expressed mRNAs and proteins by transcriptomic and proteomic comparison of frontal/temporal cortex samples from control and AD-affected subjects, followed by network analysis to predict causal interactions between different targets (mRNAs and proteins) within modules. Key driver analysis was then used to mine for the targets with a higher number of connections, which were further prioritized based on the association of mRNA expression changes with SNPs. HSPA2 emerged as one of the top hits from this approach (Table 1) and was functionally validated by showing that its overexpression could significantly increase both $A B$ and Tau/pTau levels using Tau-expressing H4 in vitro cell cultures, an observation consistent with its elevated levels in AD subjects [22].

Altogether, the studies discussed above provide an important proof of principle that different high-throughput datasets, by means of carefully crafted pipelines integrating several layers of information, may be successfully employed to predict genuine molecular drivers of cognitive impairment, even though an association between certain molecular and phenotypic traits does not formally imply a causal relationship between the associated molecules and phenotypes. Importantly, these studies also suggest that different bioinformatic pipelines can single out candidate regulators of cognitive decline that converge on shared molecular pathways (e.g., INPPL1, IGFBP5, and SEMA3F, all implicated in insulin/IGF signalling). This can significantly increase the chance of pinpointing the key pathways upstream of neuropathological events, thus facilitating the identification of optimal targets for therapeutic approaches. Although the above described studies represent 
an initial step in this direction, with several limitations related to sample type and size, to the depth of omic datasets and to downstream functional validation, they suggest potentially important roles for the molecular pathways mediated by semaphorins/ plexins, insulin/IGFs, and HSPs in the etiology of cognitive decline during normal and/or pathological aging (Fig. 1).

It is worth noting that AKT, a critical node in the insulin/ IGF pathway, can regulate the activity of the GSK3ß kinase, which plays a key role in $\mathrm{Wnt} / \mathrm{B}$-catenin-dependent signal transduction. Previous work has shown that Wnt/ß-catenindependent signalling can promote the nuclear accumulation of REST, a master transcriptional regulator of neuronal differentiation genes that also plays a protective function in cognitive decline. Nuclear REST levels in neurons of the human PFC and hippocampus increase during aging, but this effect is largely lost in cognitively impaired subjects. Functional in vitro and in vivo experiments showed that REST acts as a repressor of genes involved in cell death, $A \beta$ production, and Tau phosphorylation and that lack of REST function increases neuronal sensitivity to oxidative stress and $A ß$ and accelerates age-dependent neurodegeneration in the brain of knockout mice [23]. Based on these results, it is tempting to speculate that changes in the levels of proteins implicated in the regulation of AKT-dependent signalling and associated with cognitive decline (such as INPPL1, IGFBP5, SEMA3F) may be functionally linked, at least in part, to neuropathological phenotypes through alterations in REST activity.

The observations described above also suggest that neuropathological aging is not simply an accelerated/enhanced version of normal aging but involves the dysregulation of molecular pathways that play a protective role in healthy aging cells. This hypothesis has received strong support by a recent study that employed lateral temporal lobe samples from younger non- $A D$, elder non- $\mathrm{AD}$, and elder $\mathrm{AD}$ subjects for genome-wide profiling of histone H4 lysine 16 acetylation (H4K16ac) [24]. Three-way comparison of H4K16ac peaks in these groups of samples revealed three classes of changes: i) age-regulated changes (present both in elder non- $\mathrm{AD}$ and in elder $\mathrm{AD}$ samples, as compared to younger samples); ii) age-dysregulated changes (present only in elder non-AD samples, as compared to younger samples, but not in the elder AD group); iii) disease-specific changes (distinguishing $\mathrm{AD}$ samples from both younger and elder nonAD samples). Furthermore, H4K16ac changes between younger and elder non-AD samples negatively correlated with the changes observed between elder non-AD and $\mathrm{AD}$ samples. Parallel transcriptomic analyses showed a positive correlation between H4K16ac peak levels and the mRNA levels of nearby genes, indicating that the observed differences in H4K16ac among the

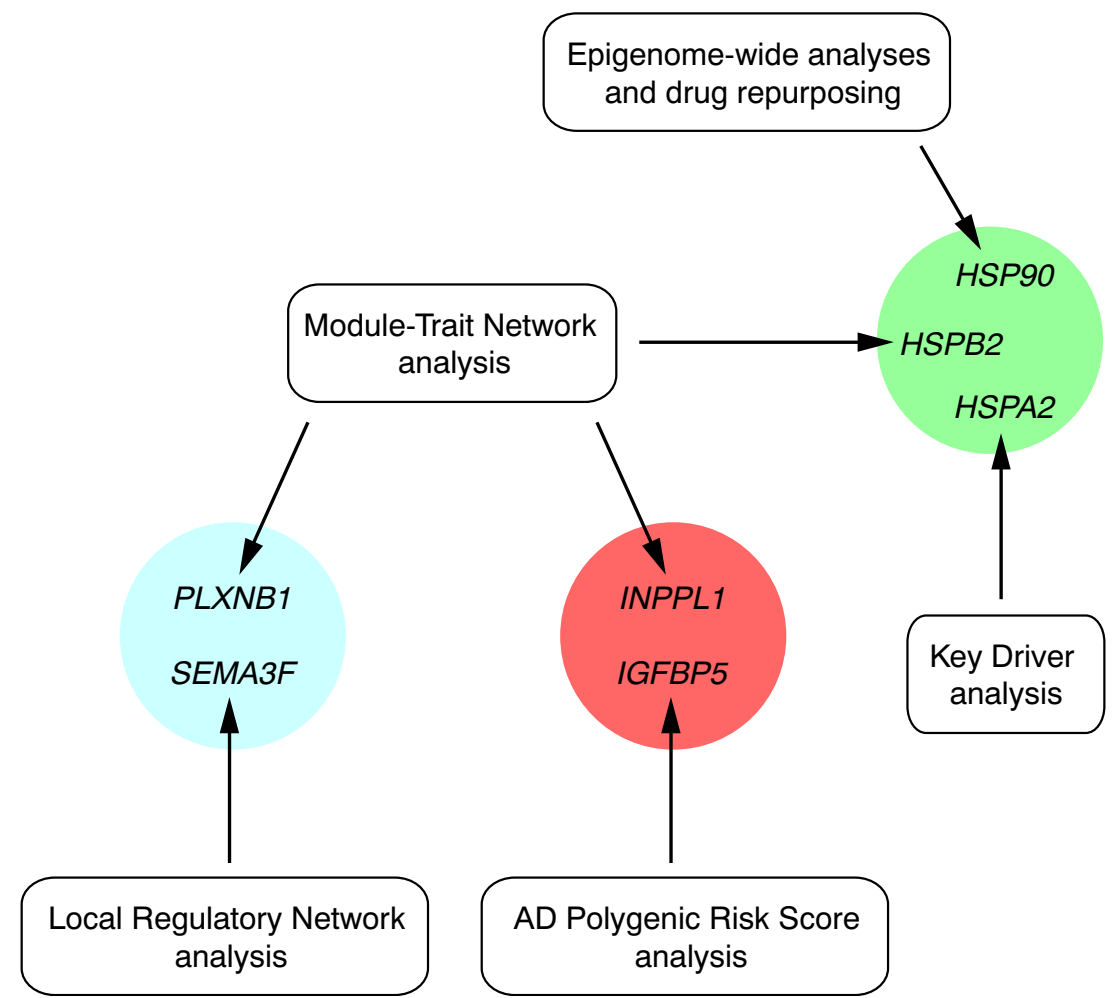

Fig. 1 Identification of candidate regulators of cognitive decline through genome-wide molecular profiling of human brain cells. The figure shows a schematic description of recent bioinformatic pipelines used to prioritize candidate genes with potential causative roles in cognitive decline, and of some of the top hits resulting from these approaches. Colored circles highlight genes identified with different strategies that are implicated in similar molecular pathways (pale blue, semaphorin/plexin signalling; red, insulin/IGF signalling; green, heat shock protein networks). See text for further details 
three groups of samples are likely to be associated with gene expression changes. Genes with decreased H4K16ac peaks in elder non-AD samples, as compared with younger samples, were enriched for REST binding motifs, thus supporting a role of REST in the regulation of aging-related transcriptional networks. Moreover, genes related to cell death regulation were the most significantly enriched category among age-dysregulated genes, in agreement with the proposed misregulation of cell deathrelated REST targets in neuropathological aging [24]. All together, these data indicate that some of the epigenetic and gene expression changes associated with normal brain aging are reversed during pathological aging, and that neuropathological conditions are also associated with specific changes that distinguish them from non-pathological aging. Pipelines designed to prioritize candidate molecular pathways for functional assays among the genes associated with age-dysregulated and disease-specific changes are expected to provide key insights into the molecular mechanisms of cognitive decline and unveil new potential targets for better therapeutic approaches.

\section{Age-Related Transcriptomic and Epigenomic Changes in Human Brain Neuronal Cells Include the Dysregulation of the Gene Networks Implicated in Synaptic Function}

The studies described in the previous section reported an association between the downregulation of gene categories related to neuronal/synaptic function and cognitive decline. For example, these categories were enriched in the modules of co-expressed genes showing a negative correlation between expression levels and cognitive impairment according to MTN analysis [15]. Moreover, according to LRN analysis, the genes predicted to be upstream of cognitive processes showed enrichment for categories linked to neurons and myelin [16]. Similar enrichment of gene categories related to neuronal function among the genes downregulated with age has been reported in other transcriptomic analyses of bulk tissue from human brain samples [25]. These observations may reflect the association of cognitive decline with the depletion of neuronal cell populations, with the dysregulation of gene expression programmes in neuronal cells, or with both these effects. To address this issue, several studies have specifically investigated the neuronal transcriptome in brain samples from aged or cognitively impaired individuals using different technical approaches.

To gain insight into the age-related gene expression changes that specifically take place in neurons, recent investigations have superimposed transcriptomic datasets of bulk brain samples from human subjects of different ages on available transcriptional signatures of seven purified murine cell types [3]. Notably, human samples were collected from ten different brain regions to characterize both region-dependent and age-associated transcriptional changes. This analysis showed that the genes differentially expressed between younger and elder samples could be classified as region-selective (i.e., the changes were specific for one or a few regions) or multi-regional (changes detectable in most or all of the tested regions). When the differentially expressed genes were also categorized based on their association with a specific cell type, neuronal-specific genes showing age-related changes resulted enriched for region-selective genes and were often downregulated with age. Nonetheless, when the analyzed samples were clustered on the basis of the expression levels of the neuronal genes, they could be mainly discriminated based on regional character rather than age, indicating that regiondependent mechanisms have a stronger influence than agerelated factors on the overall transcriptional profiles of neuronal cells. These data extend to human brain cells, and on a genomewide scale, previous results obtained in mouse neural progenitors suggesting that genes controlling positional identity, such as Hox genes, tend to maintain regional transcriptional/epigenetic patterns throughout life [26]. The same study quantified the number of NeuN-positive neurons in sections of the frontal cerebral cortex from a subset of the brain samples used for the transcriptomic assays. This analysis showed a selective decrease in neuronal cells with large cell bodies, which account for roughly $20 \%$ of the analyzed neurons, although the use of a low sample number and of a single neuronal marker represent possible caveats in these assays. Furthermore, the neuronal genes showing agerelated downregulation were enriched for specific categories related to synaptic function, ion transport, and cell death regulation [3]. These results suggest that brain aging is associated with a decrease in the expression levels of a subset of the neuronal transcriptome. This may be due to the combination of a loss of neuronal cells among selectively vulnerable neuronal subpopulations with the dysregulation of the molecular networks implicated in neuronal/synaptic activity.

Another study has superimposed transcriptomic analyses of frontal cortex samples from human subjects of different ages on a single-cell transcriptome of the mouse visual cortex to extrapolate neuronal cell type-specific transcriptional signatures in the human brain datasets and evaluate the extent of their age-related changes [27]. Although neuronal genes were generally downregulated with age, the changes were considered to be significant only for three subtype-specific gene signatures, namely those associated with Ptgs 2 -expressing, Arf5-expressing, and Batf3-expressing glutamatergic neurons of cortical layers 2-5. Additional subtype-specific signatures showed significant changes that were limited to selected gene categories. In particular, age-dependent downregulation was detected for genes related to synaptic transmission and cell-cell signalling that were also associated with Sst/ Cdk6-expressing and/or Vip/Mybpc-expressing inhibitory neurons. Finally, the authors found many genes that showed an age-related decrease in their expression levels without being associated with any particular cell type. Notably, these genes were enriched in categories related to neuronal function, such as synaptic transmission and ion membrane transport [27]. Taken together, these results support the idea that certain neuronal 
subtypes may be especially vulnerable to age-dependent cell loss, although the global neuronal population is largely preserved in the aged brain. Furthermore, they suggest that aging is associated with a dysregulation of the gene expression programmes regulating synaptic activity, which may have a stronger impact on specific subtypes, but is likely to affect the neuronal lineage in general. Nonetheless, the use of mouse transcriptomic datasets to identify human neuron-specific expression signatures represents a possible limitation of the above described studies.

Further evidence for the association of cognitive decline with the altered regulation of the synapse-related transcriptome is provided by a previous analysis of the expression levels of 340 synaptic genes in 4 different brain regions of younger non- $A D$, elder non- $\mathrm{AD}$, and elder $\mathrm{AD}$ human subjects [28]. Many of the analyzed genes were found to be differentially expressed in elder non-AD samples in comparison with younger samples, but also when comparing age-matched $\mathrm{AD}$ and non- $\mathrm{AD}$ samples, with most of the changes consisting in an age-associated or ADassociated downregulation. Notably, aging and AD mainly targeted different brain regions, as the majority of the expression changes in the non- $\mathrm{AD}$ younger-elder comparison were observed in cortical regions, whereas most of the changes between $\mathrm{AD}$ and non-AD samples were detected in the hippocampus. A linear regression analysis across all sample groups (younger, elder, $\mathrm{AD}$ ), however, revealed several genes showing progressive age-related and AD-related changes (usually a downregulation) in both cortical and hippocampal samples (i.e., the change detected in elder samples was further enhanced in $\mathrm{AD}$ samples). The genes affected by aging and $\mathrm{AD}$ were enriched for key categories related to synaptic function, such as vesicle trafficking, neurotransmitter receptors, synaptic stability/scaffolding, and neuromodulatory systems [28]. These results present some limitations, because the analyzed genes may not be entirely neuronspecific and/or synapse-specific. Moreover, transcriptional changes may not necessarily result in altered protein levels. Nevertheless, they suggest that both normal aging and neuropathological conditions may impinge on the synaptic transcriptome causing an extensive downregulation of genes implicated in key synaptic processes. The effects of aging and $\mathrm{AD}$ on the synaptic transcriptome display regional specificity, although a subset of the synaptic genes is vulnerable to the converging action of age-related and AD-dependent mechanisms. It is worth noting that, according to a follow-up study comparing the transcriptome of the same 4 brain regions in cognitively normal, mildly cognitively impaired (MCI) and AD elderly subjects, genes enriched for categories related to synaptic function, proteostasis, and mitochondrial metabolism were differentially expressed in MCI samples compared to both normal and $\mathrm{AD}$ samples. In particular, synaptic genes were often downregulated in $\mathrm{AD}$ compared to normal samples, but they were frequently upregulated in MCI samples [29]. Furthermore, a recent transcriptomic and phosphoproteomic profiling of temporal cortex samples from patients at different $\mathrm{AD}$-related pathological stages has revealed fluctuating mRNA/protein levels (down-updown patterns) of genes linked to synaptic and mitochondrial function during early phases of disease [30]. These observations suggest that the gene expression changes associated with cognitive decline, such as those in the synaptic transcriptome, follow, at least in part, non-monotonic trajectories, with trend reversals during early neuropathological stages. These observations may be critical to develop therapies intercepting cognitive impairment at its onset.

Further insight into the transcriptional patterns associated with brain aging has been gained through studies that compared the transcriptomic changes taking place in human brain samples during different temporal windows of the lifespan, such as fetal development, postnatal development, and aging [31, 32]. These analyses showed that synapse-related genes were enriched among the genes showing increases in their expression levels during both fetal and early postnatal stages, as expected from the extensive development of neural circuitries during both stages [32]. In contrast, a reversal in the trajectory of gene expression changes was detected during brain aging, because gene categories related to neuronal/synaptic function were enriched for genes upregulated during postnatal development and downregulated during aging [31]. Genes related to $\mathrm{AD}$ also tended to show up-down trends when their expression changes during fetal/ postnatal development and during aging were compared [31, 32]. Although these data are limited to the PFC [32], or the analysis did not investigate region-specific changes when samples from several brain regions were included [31], they suggest the intriguing possibility that the alterations in the neuronal/ synaptic transcriptome observed in the aged brain result, at least in part, from the dysregulation of the molecular networks regulating neural development.

Epigenomic analyses are starting to reveal the epigenetic mechanisms that underlie these age-related and diseasedependent transcriptional changes, pointing to a prominent role of histone acetylation. For example, in the above described genome-wide profiling of H4K16ac deposition in the temporal cortex from younger non- $\mathrm{AD}$, elder non- $\mathrm{AD}$ and elder $\mathrm{AD}$ subjects, the groups of genes showing age-regulated changes and disease-specific changes in the levels of H4K16ac peaks were enriched for categories related to neurons and synapses [24]. These data are consistent with the aforementioned investigation of the synaptic transcriptome, showing that both age and $\mathrm{AD}$ affect the expression of synaptic genes, with partially collaborative, but also distinct effects [28]. Furthermore, transcriptomic and genome-wide profiling of histone $\mathrm{H} 3$ lysine 27 acetylation (H3K27ac) found that, in PFC samples from human subjects of different ages, the genes downregulated in aged samples also showed reduced levels of $\mathrm{H} 3 \mathrm{~K} 27 \mathrm{ac}$ at their promoters and were enriched for categories related to neuronal and synaptic function. Notably, these patterns were observed also in the aged mouse brain and could be reversed by in vivo treatments with histone deacetylase inhibitors, such as suberoylanilide hydroxamic acid 
(SAHA), providing an initial evidence that the changes in histone acetylation may play a causative role in the age-associated dysregulation of the neuronal/synaptic transcriptome [25].

Alterations in DNA methylation may also be implicated in these transcriptional changes. By profiling DNA methylation in sorted NeuN-positive (neuronal) and NeuN-negative (nonneuronal) nuclei from the frontal/temporal cortex of non-AD and $\mathrm{AD}$ human subjects of different ages, a recent study described several hundred $\mathrm{CpGs}$ showing age-related or ADrelated methylation changes specifically in neurons, with nearby genes including categories linked to neuronal processes [33]. Although this work did not provide transcriptomic data to directly correlate DNA methylation changes with gene expression changes, studies comparing PFC samples from young and aged rats, or from age-matched cognitively unimpaired and impaired rats, demonstrated a correlation between changes in DNA methylation and mRNA levels in genes implicated in neuronal/synaptic function $[34,35]$.

The epigenomic changes in brain cells related to cognitive decline may also affect mRNA splicing besides expression levels, as shown by a recent genome-wide mapping of splicing variants in DLPFC samples from $\mathrm{AD}$ and non-AD human subjects [36], which revealed a large number of genes undergoing alternative splicing, and several genes in which splicing differences were associated with $\mathrm{AD}$ neuropathological traits. This study also found a correlation between SNPs associated with alternatively spliced genes and SNPs linked to DNA methylation/H3K9ac changes, and between splicing-related SNPs and known AD-associated SNP susceptibility variants, suggesting that some of the AD-related alternative splicing may be regulated at the epigenetic level. To identify more precisely genes showing differential splicing or transcription levels that are associated with $\mathrm{AD}$ and mediated by genetic variation, the authors performed transcriptome-wide association studies (TWAS). Notably, the genes emerging from TWAS analysis were predicted to be interconnected, at the protein level, with other known $\mathrm{AD}$ susceptibility genes, forming a AD-related network that is enriched for categories linked to endocytosis, autophagy, and lysosome function [36]. This work, however, does not distinguish RNA changes occurring upstream or downstream of neuropathological traits or representing indirect effects due to pathological alterations in other brain regions. A previous transcriptomic analysis of frontal cortex and cerebellum tissue samples and of laser-capture microdissected Purkinje neurons from human subjects of different ages found a few age-related changes that were shared between bulk tissues and Purkinje neurons, including genes implicated in endosomal/lysosomal functions [37]. This suggests that at least some of the age-associated or disease-dependent mRNA changes related to autophagic/ lysosomal processes can take place in neuronal cell types.

Given the complexity of the epigenetic regulatory mechanisms acting in neural cells [38], the studies described above are only starting to shed light on the interactions between normal and neuropathological aging processes and the neuronal epigenome, but we can expect that this will be a major avenue for future investigations.

\section{Age-Related Transcriptomic and Epigenomic Changes in Human Brain Glial Cells Include the Dysregulation of the Gene Networks Implicated in Inflammatory Responses}

Transcriptomic datasets of bulk tissue samples from the human PFC show that, opposite to the age-related downregulation of genes implicated in neuronal/synaptic function, aging and cognitive decline are associated with an increase in the expression levels of genes enriched for categories linked to immune functions and inflammation $[15,25]$. Remarkably, the genes upregulated during PFC aging, including immune/inflammation-related categories, were recently shown to be associated with a specific epigenomic signature, whereby both their promoters and gene bodies were marked by $\mathrm{H} 3 \mathrm{~K} 27 \mathrm{ac}$ deposition, unlike agedownregulated genes, in which H3K27ac peaks were found only at promoter regions. Furthermore, aging was associated with a loss of H3K27ac at gene bodies, which strongly correlated with an age-dependent increase in transcription levels. In vivo treatments of adult mice with SAHA prevented the age-related decrease of $\mathrm{H} 3 \mathrm{~K} 27 \mathrm{ac}$ at gene bodies and the transcriptional upregulation associated with this decrease, at the same time improving the cognitive performance of treated mice in specific behavioral tasks [25].

It would be crucial to extend these studies using a wider range of epigenomic analyses focused not only on fast-turnover histone acetylations like H3K9ac, H4K16ac and H3K27ac [39], but also on more stable histone acetylations such as $\mathrm{H} 3 \mathrm{~K} 4 \mathrm{ac}$ or $\mathrm{H} 3 \mathrm{~K} 56 \mathrm{ac}$ and histone methylations at $\mathrm{H} 3 \mathrm{~K} 4, \mathrm{H} 3 \mathrm{~K} 9, \mathrm{H} 3 \mathrm{~K} 27$, and H4K20, in order to evaluate the persistence and potential reversibility of age-related signatures. Notwithstanding these caveats, the above described results suggest the existence of an epigenomic mechanism controlling the activation levels of the brain inflammatory transcriptome, which may be prone to an age-related dysregulation predisposing the brain to increased inflammatory responses and cognitive impairment.

Inflammatory responses in the brain are mainly mediated by resident glial populations, especially astrocytes and microglia. In agreement with this notion, genome-wide transcriptomic analyses of the cell type-specific and the region-specific gene expression changes associated with human brain aging found that the genes showing multi-regional changes (i.e., detected across most of the tested brain areas) were often upregulated, microglialspecific and enriched for categories related to immune response. Consequently, in contrast to neuronal gene expression, microglial transcriptional signatures mainly clustered the analyzed samples according to age, rather than regional identity, indicating that the age-related transcriptional response of microglia generally lacks a specific regional character, with the exception of the cerebellum 
[3]. Therefore, aging is associated with a widespread increase of immune-related gene expression in microglial cells, which is likely to be functionally linked to the inflammatory environment of the aged brain.

Two recent studies have profiled the transcriptome of human microglial cells that were sorted either from the DLPFC [40] or from the parietal cortex [41]. The DLPC dataset was derived from elderly subjects, some of them with a diagnosis of $\mathrm{AD}$, whereas the parietal cortex dataset was obtained from cognitively normal subjects of various ages. Both studies confirmed that the human microglia transcriptome is differentially regulated with age. In particular, the analysis of gene expression levels in relation to sample age in the parietal cortex dataset revealed several hundred genes that were differentially expressed in younger and elder microglia and that were enriched for categories related to immune response, cytoskeleton, and cell adhesion [41]. A large number of age-related transcriptional changes were also found by comparing the DLPFC dataset with previous microglia signatures obtained from middle-aged subjects. The genes upregulated in the aged DLPFC microglia were enriched for categories related to amyloid fiber formation, immune functions, and endosomal/vacuolar pathways [40].

A substantial group of genes preferentially expressed in the aged human microglia, named HuMi_Aged gene set, was identified by superimposing the microglial-specific DLPFC profiles with the transcriptome of bulk DLPFC tissue and selecting the genes robustly enriched in sorted cells compared to tissue samples. This set contained several known susceptibility genes for late onset $\mathrm{AD}$, although the sample number used for microglia purification was too small to find significant associations between AD-related neuropathological traits in these samples and HuMi_Aged genes [40]. A recent study combined a morphological evaluation of the proportion of active microglia (PAM) in postmortem cortical samples from elderly subjects with GWAS to correlate genomic variation with microglia activation and $\mathrm{AD}$. These analyses revealed an association of PAM-related and ADrelated susceptibility variants, suggesting that the mechanisms leading to increased microglia activation in the aged brain have a genotypic component, which also seems to predispose to cognitive impairment [42]. All together, the observations described above suggest that the inflammatory transcriptome of microglia cells is broadly hyperactivated in the aging brain, that both genetic variation and epigenetic regulation contribute to this agerelated upregulation, and that the genetic and epigenetic factors linked to increased microglia activation may contribute to cognitive decline.

Unlike the microglia-specific transcriptome, which appears to be mainly affected by age with limited regional differences, astrocyte-specific genes in the human brain show complex patterns that are dependent on both regional identity and age, as shown by superimposing transcriptomic profiling of bulk human brain tissues to mouse brain single-cell datasets [3]. In particular, these genes reveal a significant degree of regional diversity in the younger brain, clearly separating the transcriptional profiles of different brain areas, such as the cerebral cortex, the hippocampus, the cerebellum, and the brainstem. Aging brings about marked shifts in the regional character of astrocytic gene expression, causing loss of regional differences in the transcriptional profiles of the aged brain [3]. These age-related changes may be causally relevant to the alterations in the neural circuitry underlying cognitive decline, given the extensive functional interactions between astroglial and neuronal cell populations. In support of this hypothesis, recent transcriptomic profiling of purified astrocytes from young and aged mice showed that aged astrocytes upregulated genes involved in synaptic elimination and neuronal damage, such as those encoding for key components of the complement cascade [43]. Given the significant differences found in the transcriptome of purified human and mouse microglia [41, 44], however, the age-related changes identified in aged mice might not faithfully recapitulate the alterations in the aged human astroglia. Therefore, analyses performed with purified astrocyte cell populations from human brain samples are necessary to fill this knowledge gap.

\section{Conclusions and Future Perspectives}

The last couple of years have witnessed a major leap forward in employing human postmortem tissue samples as a tractable experimental system to elucidate the molecular changes associated with human brain aging and to gain insight into the possible mechanisms causing cognitive decline during normal and neuropathological aging. This breakthrough has been made possible by improved and affordable NGS platforms allowing the generation of large molecular datasets, which are amenable to mathematical and computational approaches to predict the likelihood of causal relationships based on statistical associations among diverse molecular and phenotypic traits. Thus, although functional studies are extremely difficult to do in human subjects, mechanistic predictions can be made based on human datasets and subsequently validated in other experimental paradigms.

The experimental use of human brain samples is inherently exposed to potentially confounding factors such as the extent of postmortem tissue preservation and the degree to which the general population is represented in the cohort used for sampling. Besides these obvious caveats, several challenges will have to be faced to improve the power of molecular investigations of human brain aging. First, a better temporal resolution of the age-dependent changes will need to be achieved, with a stronger focus on the early onset of brain aging processes and cognitive decline. Second, it will be essential to perform molecular profiling of purified neurons and astrocytes from human brain samples, as already done with microglia, and to implement single-cell approaches to dissect the agerelated changes taking place in specific cell subpopulations. Third, it will be important to extend epigenomic analyses by 
the profiling of additional chromatin modifications, and to focus on the age-associated changes in the non-coding transcriptome. The modifications in gene expression levels detected during normal or pathological brain aging may also reflect alterations in transcript stability, which may be regulated by microRNAs [45]. In agreement with this hypothesis, agerelated modulation of microRNA levels in the rodent brain has been reported [46, 47]. Notably, circular RNAs have been shown to accumulate in the aging brain [48], and long noncoding RNAs are also implicated in various aging processes [49]. Finally, although pluripotent cells and organoid culture methods have allowed to perform functional assays in human in vitro neural cell systems [50], mouse genetic models will remain essential for functional studies in vivo in the foreseeable future. Therefore, more work is crucially needed to clarify the conserved and the divergent aspects in the genomewide mechanisms underlying brain aging in humans and in animal model systems.

Shortly after this review was completed, Mathys and coauthors have reported a single-cell transcriptomic analysis of the human PFC using postmortem samples from subjects with varying degrees of $\mathrm{AD}$ pathology[51].

Required Author Forms Disclosure forms provided by the authors are available with the online version of this article.

\section{References}

1. Morrison JH, Hof PR (1997) Life and death of neurons in the aging brain. Science 278:412-419

2. Morrison JH, Hof PR (2007) Life and death of neurons in the aging cerebral cortex. Int Rev Neurobiol 81:41-57. https://doi.org/10. 1016/S0074-7742(06)81004-4

3. Soreq L, UK Brain Expression Consortium, North American Brain Expression Consortium, et al (2017) Major Shifts in Glial Regional Identity Are a Transcriptional Hallmark of Human Brain Aging. Cell Rep 18:557-570. https://doi.org/10.1016/j.celrep.2016.12.011

4. Kennard JA, Brown KL, Woodruff-Pak DS (2013) Aging in the cerebellum and hippocampus and associated behaviors over the adult life span of CB6F1 mice. Neuroscience 247:335-350. https://doi.org/10.1016/j.neuroscience.2013.06.002

5. Calì C, Wawrzyniak M, Becker C, et al (2018) The effects of aging on neuropil structure in mouse somatosensory cortex-A 3D electron microscopy analysis of layer 1 . PloS One 13:e 0198131. https://doi. org/10.1371/journal.pone.0198131

6. Eavri R, Shepherd J, Welsh CA, et al (2018) Interneuron Simplification and Loss of Structural Plasticity As Markers of Aging-Related Functional Decline. J Neurosci Off J Soc Neurosci 38:8421-8432. https://doi.org/10.1523/JNEUROSCI.0808-18.2018

7. Fattoretti P, Malatesta M, Cisterna B, et al (2018) Modulatory Effect of Aerobic Physical Activity on Synaptic Ultrastructure in the Old Mouse Hippocampus. Front Aging Neurosci 10:141. https://doi. org/10.3389/fnagi.2018.00141

8. Mostany R, Anstey JE, Crump KL, et al (2013) Altered synaptic dynamics during normal brain aging. J Neurosci Off J Soc
Neurosci 33:4094-4104. https://doi.org/10.1523/JNEUROSCI. 4825-12.2013

9. Di Benedetto S, Müller L, Wenger E, et al (2017) Contribution of neuroinflammation and immunity to brain aging and the mitigating effects of physical and cognitive interventions. Neurosci Biobehav Rev 75:114-128. https:// doi.org/10.1016/j.neubiorev.2017.01.044

10. Liang KJ, Carlson ES (2019) Resistance, vulnerability and resilience: A review of the cognitive cerebellum in aging and neurodegenerative diseases. Neurobiol Learn Mem. https://doi.org/10. 1016/j.nlm.2019.01.004

11. Lupo G, Nisi PS, Esteve P, et al (2018) Molecular profiling of aged neural progenitors identifies Dbx2 as a candidate regulator of ageassociated neurogenic decline. Aging Cell 17:e12745. https://doi. org/10.1111/acel.12745

12. Lupo G, Gioia R, Nisi PS, et al (2019) Molecular Mechanisms of Neurogenic Aging in the Adult Mouse Subventricular Zone. J Exp Neurosci 13:1179069519829040. https://doi.org/10.1177/ 1179069519829040

13. Shi Z, Geng Y, Liu J, et al (2018) Single-cell transcriptomics reveals gene signatures and alterations associated with aging in distinct neural stem/progenitor cell subpopulations. Protein Cell 9:351364. https://doi.org/10.1007/s13238-017-0450-2

14. Lodato MA, Rodin RE, Bohrson CL, et al (2018) Aging and neurodegeneration are associated with increased mutations in single human neurons. Science 359:555-559. https://doi.org/10.1126/ science.aao4426

15. Mostafavi S, Gaiteri C, Sullivan SE, et al (2018) A molecular network of the aging human brain provides insights into the pathology and cognitive decline of Alzheimer's disease. Nat Neurosci 21:811819. https://doi.org/10.1038/s41593-018-0154-9

16. Tasaki S, Gaiteri C, Mostafavi S, et al (2018) Multi-omic Directed Networks Describe Features of Gene Regulation in Aged Brains and Expand the Set of Genes Driving Cognitive Decline. Front Genet 9:294. https://doi.org/10.3389/fgene.2018.00294

17. Tasaki S, Gaiteri C, Mostafavi S, et al (2018) The Molecular and Neuropathological Consequences of Genetic Risk for Alzheimer's Dementia. Front Neurosci 12:699. https://doi.org/10.3389/fnins. 2018.00699

18. Klein H-U, McCabe C, Gjoneska E, et al (2019) Epigenome-wide study uncovers large-scale changes in histone acetylation driven by tau pathology in aging and Alzheimer's human brains. Nat Neurosci 22:37-46. https://doi.org/10.1038/s41593-018-0291-1

19. De Jager PL, Srivastava G, Lunnon K, et al (2014) Alzheimer's disease: early alterations in brain DNA methylation at ANK1, BIN1, RHBDF2 and other loci. Nat Neurosci 17:1156-1163. https://doi.org/10.1038/nn.3786

20. Yu L, Petyuk VA, Gaiteri C, et al (2018) Targeted brain proteomics uncover multiple pathways to Alzheimer's dementia. Ann Neurol 84:78-88. https://doi.org/10.1002/ana.25266

21. Nakayama H, Bruneau S, Kochupurakkal N, et al (2015) Regulation of mTOR Signaling by Semaphorin 3F-Neuropilin 2 Interactions In Vitro and In Vivo. Sci Rep 5:11789. https://doi. org/10.1038/srep11789

22. Petyuk VA, Chang R, Ramirez-Restrepo M, et al (2018) The human brainome: network analysis identifies HSPA2 as a novel Alzheimer\&rsquo;s disease target. Brain J Neurol 141:27212739. https://doi.org/10.1093/brain/awy215

23. Lu T, Aron L, Zullo J, et al (2014) REST and stress resistance in ageing and Alzheimer's disease. Nature 507:448-454. https://doi. org $/ 10.1038 /$ nature 13163

24. Nativio R, Donahue G, Berson A, et al (2018) Dysregulation of the epigenetic landscape of normal aging in Alzheimer's disease. Nat Neurosci 21:497-505. https://doi.org/10.1038/s41593-018-0101-9

25. Cheng H, Xuan H, Green CD, et al (2018) Repression of human and mouse brain inflammaging transcriptome by broad gene-body 
histone hyperacetylation. Proc Natl Acad Sci U S A 115:76117616. https://doi.org/10.1073/pnas.1800656115

26. Carucci N, Cacci E, Nisi PS, et al (2017) Transcriptional response of Hoxb genes to retinoid signalling is regionally restricted along the neural tube rostrocaudal axis. R Soc Open Sci 4:160913. https:// doi.org/10.1098/rsos.160913

27. French L, Ma T, Oh H, et al (2017) Age-Related Gene Expression in the Frontal Cortex Suggests Synaptic Function Changes in Specific Inhibitory Neuron Subtypes. Front Aging Neurosci 9: 162. https://doi.org/10.3389/fnagi.2017.00162

28. Berchtold NC, Coleman PD, Cribbs DH, et al (2013) Synaptic genes are extensively downregulated across multiple brain regions in normal human aging and Alzheimer's disease. Neurobiol Aging 34: 1653-1661. https://doi.org/10.1016/j.neurobiolaging.2012.11.024

29. Berchtold NC, Sabbagh MN, Beach TG, et al (2014) Brain gene expression patterns differentiate mild cognitive impairment from normal aged and Alzheimer's disease. Neurobiol Aging 35:19611972. https://doi.org/10.1016/j.neurobiolaging.2014.03.031

30. Marttinen M, Paananen J, Neme A, et al (2019) A multiomic approach to characterize the temporal sequence in Alzheimer's disease-related pathology. Neurobiol Dis 124:454-468. https://doi. org/10.1016/j.nbd.2018.12.009

31. Dönertaș HM, İzgi H, Kamacıoğlu A, et al (2017) Gene expression reversal toward pre-adult levels in the aging human brain and agerelated loss of cellular identity. Sci Rep 7:5894. https://doi.org/10. 1038/s41598-017-05927-4

32. Colantuoni C, Lipska BK, Ye T, et al (2011) Temporal dynamics and genetic control of transcription in the human prefrontal cortex. Nature 478:519-523. https://doi.org/10.1038/nature10524

33. Gasparoni G, Bultmann S, Lutsik P, et al (2018) DNA methylation analysis on purified neurons and glia dissects age and Alzheimer's disease-specific changes in the human cortex. Epigenetics Chromatin 11:41. https://doi.org/10.1186/s13072-018-0211-3

34. Ianov L, Riva A, Kumar A, Foster TC (2017) DNA Methylation of Synaptic Genes in the Prefrontal Cortex Is Associated with Aging and Age-Related Cognitive Impairment. Front Aging Neurosci 9: 249. https://doi.org/10.3389/fnagi.2017.00249

35. Ianov L, Rani A, Beas BS, et al (2016) Transcription Profile of Aging and Cognition-Related Genes in the Medial Prefrontal Cortex. Front Aging Neurosci 8:113. https://doi.org/10.3389/ fnagi.2016.00113

36. Raj T, Li YI, Wong G, et al (2018) Integrative transcriptome analyses of the aging brain implicate altered splicing in Alzheimer's disease susceptibility. Nat Genet 50:1584-1592. https://doi.org/10. 1038/s41588-018-0238-1

37. Kumar A, Gibbs JR, Beilina A, et al (2013) Age-associated changes in gene expression in human brain and isolated neurons. Neurobiol Aging 34:1199-1209. https://doi.org/10.1016/j.neurobiolaging. 2012.10.021

38. Cacci E, Negri R, Biagioni S, Lupo G (2017) Histone Methylation and microRNA-dependent Regulation of Epigenetic Activities in
Neural Progenitor Self-Renewal and Differentiation. Curr Top Med Chem 17:794-807

39. Zheng Y, Thomas PM, Kelleher NL (2013) Measurement of acetylation turnover at distinct lysines in human histones identifies long-lived acetylation sites. Nat Commun 4:2203. https://doi.org/ 10.1038/ncomms3203

40. Olah M, Patrick E, Villani A-C, et al (2018) A transcriptomic atlas of aged human microglia. Nat Commun 9:539. https://doi.org/10. 1038/s41467-018-02926-5

41. Galatro TF, Holtman IR, Lerario AM, et al (2017) Transcriptomic analysis of purified human cortical microglia reveals age-associated changes. Nat Neurosci 20:1162-1171. https://doi.org/10.1038/nn.4597

42. Felsky D, Roostaei T, Nho K, et al (2019) Neuropathological correlates and genetic architecture of microglial activation in elderly human brain. Nat Commun 10:409. https://doi.org/10.1038/ s41467-018-08279-3

43. Boisvert MM, Erikson GA, Shokhirev MN, Allen NJ (2018) The Aging Astrocyte Transcriptome from Multiple Regions of the Mouse Brain. Cell Rep 22:269-285. https://doi.org/10.1016/j. celrep.2017.12.039

44. Grabert K, Michoel T, Karavolos MH, et al (2016) Microglial brain region-dependent diversity and selective regional sensitivities to aging. Nat Neurosci 19:504-516. https://doi.org/10.1038/nn.4222

45. Jonas S, Izaurralde E (2015) Towards a molecular understanding of microRNA-mediated gene silencing. Nat Rev Genet 16:421-433. https://doi.org/10.1038/nrg3965

46. Inukai S, de Lencastre A, Turner M, Slack F (2012) Novel microRNAs differentially expressed during aging in the mouse brain. PloS One 7:e40028. https://doi.org/10.1371/journal.pone. 0040028

47. Yin L, Sun Y, Wu J, et al (2015) Discovering novel microRNAs and age-related nonlinear changes in rat brains using deep sequencing. Neurobiol Aging 36:1037-1044. https://doi.org/10.1016/j. neurobiolaging.2014.11.001

48. Knupp D, Miura P (2018) CircRNA accumulation: A new hallmark of aging? Mech Ageing Dev 173:71-79. https://doi.org/10.1016/j. mad.2018.05.001

49. Xing W, Gao W, Mao G, et al (2017) Long non-coding RNAs in aging organs and tissues. Clin Exp Pharmacol Physiol 44 Suppl 1: 30-37. https://doi.org/10.1111/1440-1681.12795

50. Lupo G, Bertacchi M, Carucci N, et al (2014) From pluripotency to forebrain patterning: an in vitro journey astride embryonic stem cells. Cell Mol Life Sci CMLS 71:2917-2930. https://doi.org/10. 1007/s00018-014-1596-1

51. Mathys H, Davila-Velderrain J, Peng Z, et al (2019) Single-cell transcriptomic analysis of Alzheimer's disease. Nature. https://doi. org/10.1038/s41586-0191195-2

Publisher's Note Springer Nature remains neutral with regard to jurisdictional claims in published maps and institutional affiliations. 